\title{
ESTRATÉGIA REPRODUTIVA E ALGUNS ASPECTOS DEMOGRÁFICOS DO CAMARÃO Palaemonetes carteri GORDON,1935 NA AMAZONIA CENTRAL, RIO NEGRO.
}

\author{
Olga O. COLLART', Adriana ENRICONI'.
}

RESUMO - A ecologia do camarão Palaemonetes carteri Gordon, 1935 (Decapoda: Palaemonidae) foi estudada através de coletas mensais, realizadas de janeiro 1989 até maio 1990 , na região de Manaus. $P$. carteri vive na liteira submersa dos igarapés de terra firme em áreas baixas inundadas periodicamente pelas enchentes dos rios. A estratégia de reprodução e a dinâmica da razão sexual foram estudados na base dos 4,441 indivíduos coletados. $P$. carteri mostrou um dimorfismo sexual do comprimento, as fêmeas sendo maiores que os machos. $O$ periodo de reprodução foi restrito a enchente e a cheia das águas. O número de ovos por fêmea variou entre 5 e 39 , sem nenhuma correlação com o tamanho das fêmeas. A razão sexual mostrou uma predominância de fêmeas sem nenhuma variação sazonal significativa.

Palavras-chave: Palaemonetes carteri, ecologia, estratégia reprodutiva, Amazônia central, Palaemonidae.

Reproductive Strategy and Demographic Traits of the Prawn Palaemonetes carteri Gordon, 1935 in Central Amazon, rio Negro.

ABSTRACT - The ecology of the prawn Palaemonetes carteri Gordon,1935(Decapoda: Palaemonidae) was studied through field collections realized in Central Amazon, in the vicinities of Manaus. P. carteri inhabits the submerged litter of small forest streams submitted to annual flooding. From January 1989 to May 1990, 4,441 individuals were found. Reproductive strategy, some demographic traits and sex ratio fluctuations were investigated. $P$. carteri showed sexual dimorphism in size, females being bigger than males. The reproductive period was restricted to rising and high waters. Females carried 5 to 39 large eggs. There was no significant correlation between clutch size and females carapace length. The sex ratio of adults was ca 0.60 without significant seazonal variations.

Key-words: Palaemonetes carteri, ecology, reproduction, Central Amazon, Palaemonidae.

\section{INTRODUÇÃO}

O camarão Palaen:onetes carteri é o mais comum das quatro espécies do gênero Palaemonetes encontradas na America do Sul. A sua localidade tipo é o rio Cuyuni, na Guiana Inglesa, onde ele foi descrito por Gordon em 1935. Ele se encontra em abundância na Venezuela, nas três Guianas (HOLTHUIS, 1952), a na bacia do rio
Amazonas até o Mato Grosso do Sul (rio Tapajós, rio Madeira, rio Guaporé e rio Ucayali). P. carteri concentra-se nas áreas marginais dos rios denominadas ria-lake, assim como na liteira submersa dos igarapés de terra firme, em áreas baixas inundadas periodicamente pelas enchentes dos rios. Os trabalhos publicados sobre $P$. carteri apresentam apenas dados relativos a levantamento faunístico e

1 Laboratório de Carcinologia, ANTENNE ORSTOM/INPA, Caixa Postal 478, 69011-970, Manaus, Amazonas, Brasil. 
sistemática (HOLTHUIS, 1952: GOMEZ-CORREA,

RODRIGUEZ, 1981;

1977 ;

KENSLEY \& WALKER, 1982; COELHO \& RAMOS-PORPO, 1985 ; WALKER \& FERREIRA, 1985; PEREIRA, 1986; RAMOS-PORTO \& COELHO, 1990; WALKER, 1992).

Raramente foram analisados dados relativos a ecologia desta espécie (ENRICONI \& ODINETZ COLLART, 1992: ODINETZ COLLART \& MAGALHÃES, 1992). Entretanto, MAGALHÃES (1986a) descreveu o desenvolvimento larval abreviado da espécie-irmã simpátrica. Palaemonetes ivonicus.

Este trabalho apresenta a estratégia de reprodução de $P$. carter $r$, relacionando varias características demográficas do camarão com os fatores ambientais do meio aquático.

\section{ÁREA DE ESTUdO}

As coletas foram realizadas na região de Manaus, em vários igarapés próximo à desembocadura com o Rio Negro (Fig. 1). Os locais de coleta foram agrupados em duas amostras: (1) referente ao baixo Tarumãzinho, área típica de drenagem de igarapés de terra firme na margem esquerda do Rio Negro e (2) referente ao Furo Grande, na margem direita do Rio Negro, perto da confluência com o rio Solimões. Foram assim consideradas como amostra da área 1 , as coletas realizadas no furo, igarapé e lago do Acural, no igarapé do Mari, no igarapé do Tupé e no lago do Arrombado. A amostra da área 2 é proveniente dos igarapés do Camarão, do Alagadiço, do Cabeçudo e do Morcego, assim como do Furo Grande.

Todos os locais de coleta sofrem inundações anuais decorrentes do ciclo hidrológico do Rio Negro e do Rio Solimões, e apresentam características de ria-lake, ou seja de áreas baixas na margem do rio inundadas periodicamente pelas enchentes (área 1), ou de alagado permanente (área 2). A área 1 esta sob a influência direta das águas do Rio Negro, pobres em nutrientes e caracterizadas por um $\mathrm{pH}$ ácido $(4.5$ $5,5)$, enquanto a área 2, sofre, durante a enchente e a cheia. a influência do rio Solimões, caracterizado por um $\mathrm{pH}$ mais alto (6.0-6.9) e uma alta carga sedimentar (ODINETZ COLLART \& MOREIRA, 1989).

Durante o periodo de estudo. o ciclo hidrológico do Rio Negro apresentou as fases seguintes (Fig. 2A):

- enchente de dezembro até maiode 89 , com o repiquete em fevereiro;

- cheia de junho a agosto de 89;

- vazante rápida até outubro.

- seca prolongada de outubro de89 até fevereiro de 90 , com repiquete em dezembro.

- enchente de março até maio de 90.

\section{MATERIAL E MÉTODOS}

Coletas mensais foram realizadas de dia e de noite, de janeiro de 89 até maio de 90 . Foi empregado um rapiché feito com um retângulo de ferro de 


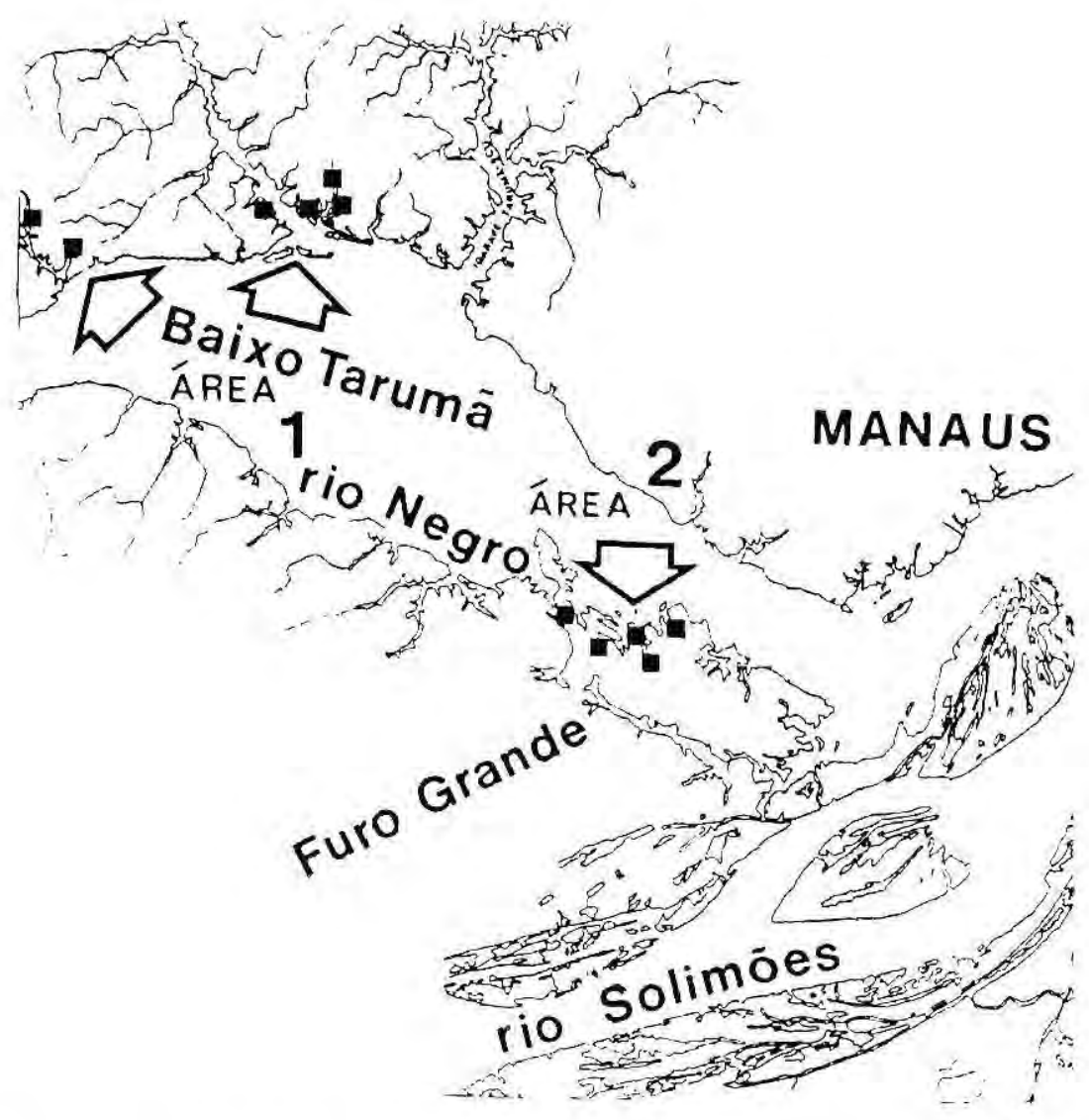

Figura 1. Área de estudo na Amazônia Central: baixo Tarumãzinho (área 1) e Furo Grande (área 2), perto da confluência do rio Negro com o rio Solimões

$30 \times 40 \mathrm{~cm}$, adaptado a uma rede de nylon de $1 \mathrm{~mm}$ de abertura de malha, presa à armação e fixado a uma aste de $1,50 \mathrm{~m}$, a qual é mergulhada na água, levantando toda a liteira do fundo, de 30 a $80 \mathrm{~cm}$ de profundidade. Após a triagem, feita em uma bandeja comum, os indivíduos são conservados em álcool $70 \%$.

No laboratório, cada amostra foi analisada por local de coleta. Cada individuo foi medido (comprimento cefalotorácico), e seu sexo determina- do (observação do segundo par de pleópodos) em lupa binocular. $\mathrm{O}$ número de ovos foi contado em 300 fềmeas ovadas, retirando-se com pinça os pleópodos, aos quais eles estão aderidos. A caracterização sistemática foi feita na base da análise morfológica de 676 indivíduos, onde:

- mediu-se o comprimento cefalotorácico, da margem posterior da cavidade orbital até o ponto distal do cefalotórax;

- contou-se o número de dentes 


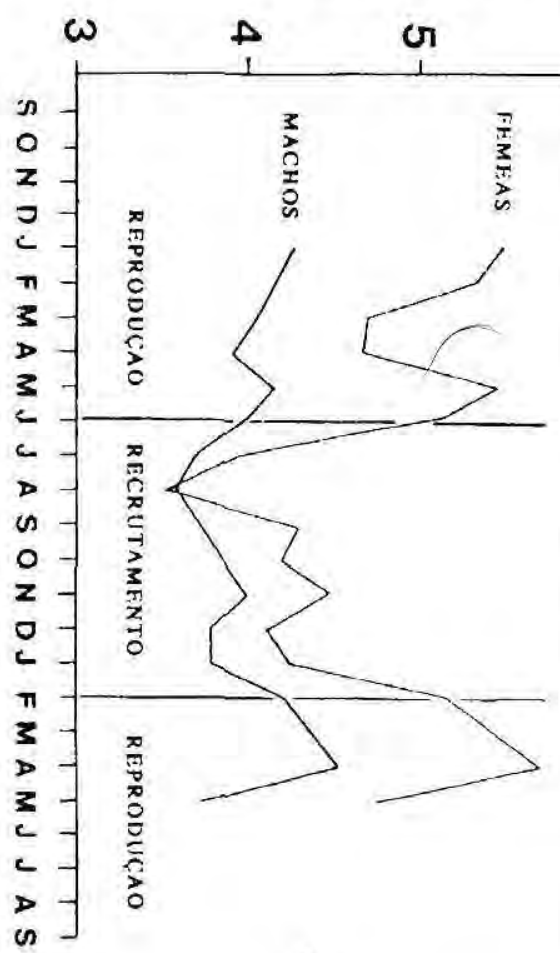

A

$$
\text { a) }
$$

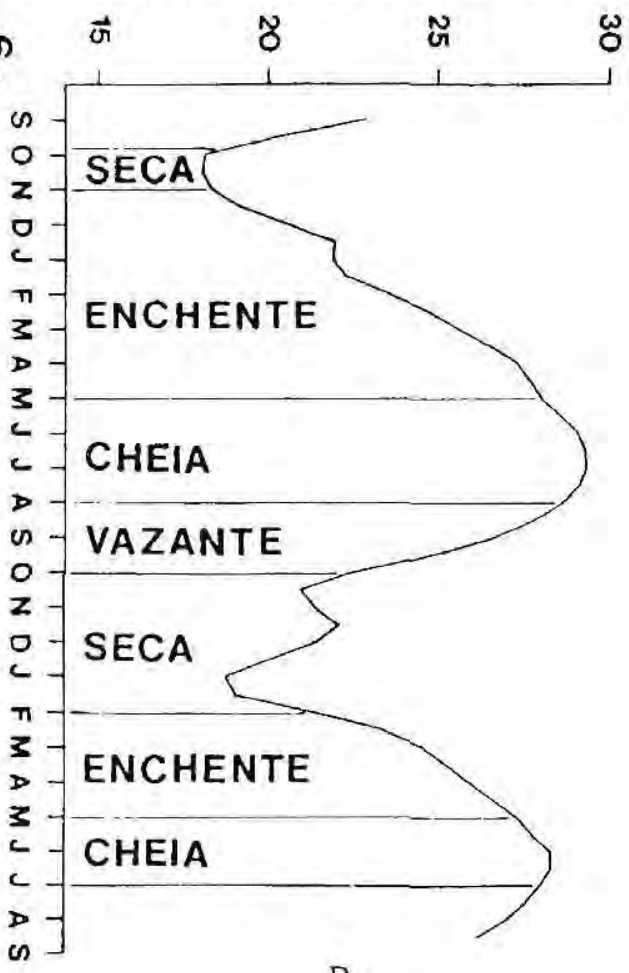

B

Figura 2. A) Evolução sazonal do nivel da água do rio Negro (medido no porto de Manaus) em 1989-1990: B) Variação mensal do comprimento médio dos machos e das fêmeas de $P$. carteri.

na margem superior e inferior do rostro;

- observou-se a posição da espinha branquiostegal em relação a margem anterior do cefalotórax.

O estágio de maturação dos ovários foi determinado por caracterização macroscópica, observando em lupa binocular o diâmetro dos ovócitos, assim como a cor, a textura e a localização dos ovários de cada fêmea, após preservação em álcool $70 \%$.

A determinação dos estágios de maturação foi estabelecida baseada na classificação de SUBRAHMANIAM (1965) com as seguintes relações: estágio 1: ovário incolor, muito reduzido com textura granulosa fina;

estágio 2: gônadas opacas visíveis através da carapaça, recobrindo parte do estômago até a metade do cefalotórax;

estágio 3: gônadas opacas amareladas visíveis através da carapaça, recobrindo parte do estômago até o terço anterior do cefalotórax; ovócitos bem distintos com diâmetro até $0,42 \mathrm{~mm}$;

estágio 4: gônadas opacas amarelas visíveis através da carapaça, preenchendo toda a cavidade cefalotorácica dorsal até a base do rostro; ovócitos bem distintos com diâmetro de $0.42 \mathrm{~mm}$ até $0,84 \mathrm{~mm}$; 
estágio 5: fềmea ovada com ovos fixados nos pleópodos

estágio 6: fềmea após desova com vácuo na cavidade cefalotorácica dorsal.

A análise de variância não paramétrica de Freedmann foi feita para testar um padrão na variação sazonal da razão sexual dos camarões considerando as duas coletas como réplicas independentes.

\section{RESULTADOS}

\section{Caracterização sistemática}

O camarão Palaemonetes carteri possui um rostro fino, curvado para cima na parte distal, alcançando a ponta do escafocérito. A margem superior do rostro apresentou geralmente 6,7 ou 8 dentes, raramente 5 ou 9 , com 1 dente pós-orbital: a margem inferior apresentou geralmente 3 ou 4 dentes, as vezes 5 e raramente 2 ou 6 (Tab. 1). No total, $94,82 \%$ dos individuos apresentaram um rostro com 6-8 dentes na margem superior e 3-5 dentes na margem inferior:

A ponta anterior do espinho branquiostegal foi localizada no mesmo nível que a margem anterior do cefalotórax em $78,10 \%$ dos indivíduos, ultrapassando à margem em $8.43 \%$ dos indivíduos, e nitidamente posterior a margem do cefalotórax em $13,47 \%$ dos camarões.

\section{Distribuição do comprimento}

Palaemonetes carteri foi observado em todos os locais de coleta durante todo ano. Porém, um número maior de individuos foi coletado na área $2(\mathrm{~N}=3609)$, perto da confluência do rio Negro com o rio Solimões, que nas águas pretas da área I $(\mathrm{N}=832)$, independentemente da época do ano.

O comprimento cefalotorácico global da populaçao foi de $4.50 \mathrm{~mm}$ na área 1 e de $4.62 \mathrm{~mm}$ na área 2 . As fêmeas foram maiores que os machos em ambas as áreas o ano todo (Fig. 2B). Ambos os sexos apresentaram uma variação sazonal do comprimento: os maiores indivíduos foram observados de janeiro à junho 1989 , e de fevereiro à maio 90 , durante a enchente das águas, enquanto os camarões de comprimento menor foram observados durante a vazante e a seca, de julho 89 até janeiro 90 (Fig. 2B).

\section{Dinâmica da razão sexual}

A razão sexual mostrou uma predominância significativa de fềmeas na população (Fig. $3 \mathrm{~A}$ e B):

- a razão sexual foi de $60.94 \%$ na área 1 e $58.38 \%$ na área 2 ;

- a razão sexual média, que considera os valores de todas as amostras mensais, foi de $70.82 \%$ na área I e $64.04 \%$ na área 2.

Considerando os dois locais de coleta como réplicas independentes, a razão sexual apresentou um padrão de variação sazonal estatisticamente significativo (teste de Freedman:

$$
\mathrm{X} 2=32.54 ; \mathrm{P}<0.001 ; \mathrm{gl}=10) \text { : em }
$$
ambas as áreas, a percentagem de fêmeas foi maior de junho até setembro, assim como em dezembro (Fig. 4). 


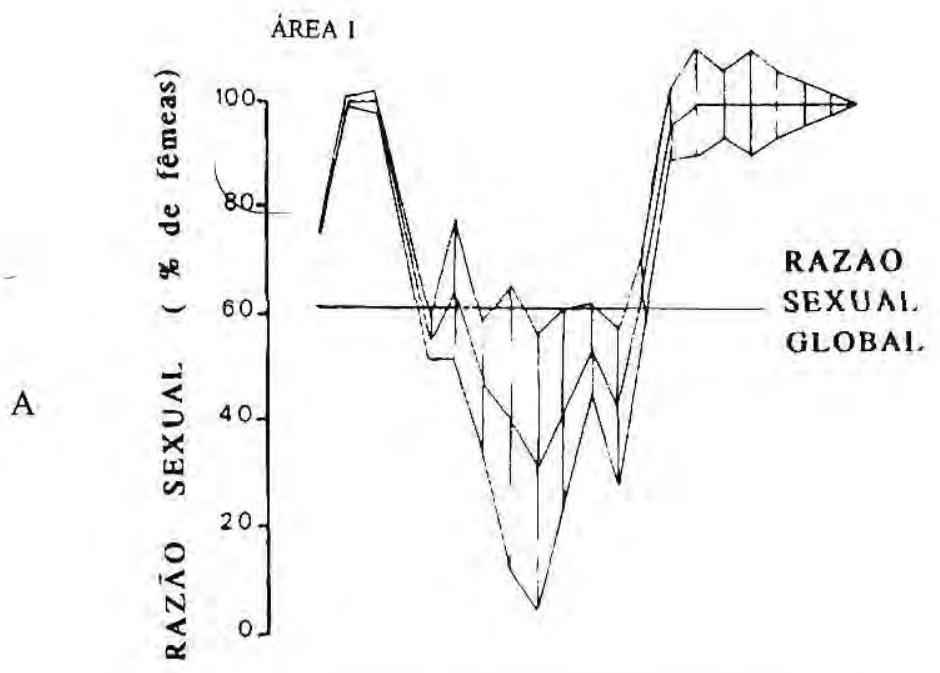

B

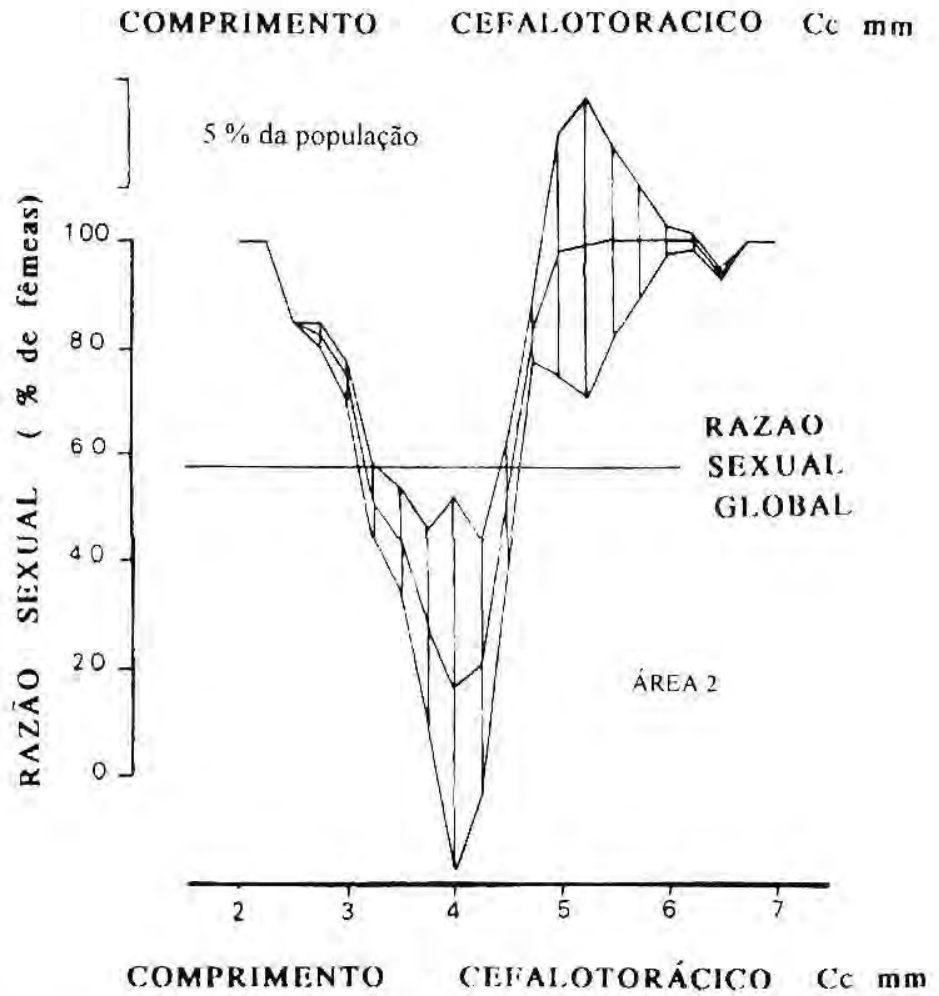

Figura 3. Distribuição das frequências de fêmeas na população de $P$. carteri em função do comprimento: A) no baico Tarumãzinho (área 1) e B) no Furo Grande (área 2). 


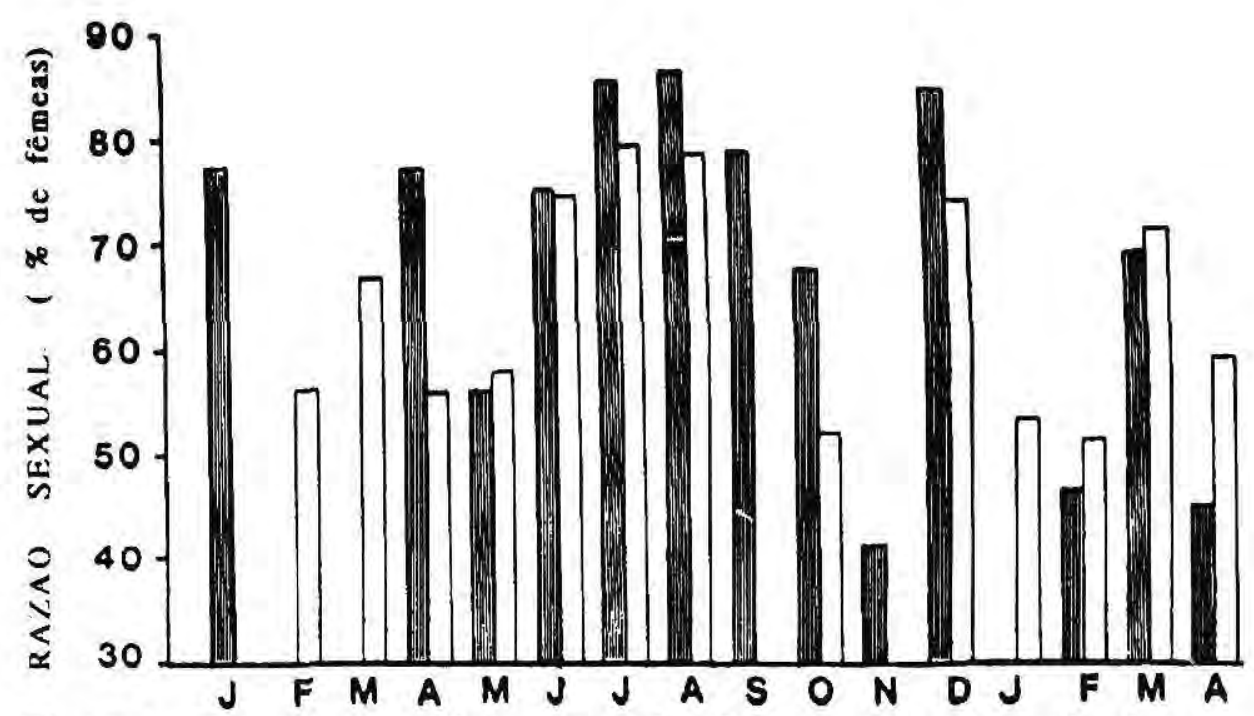

Figura 4. Evolução sazonal da razão sexual de Palaemaneteri carteri coletado na área 1, Baixo Tarumã Giriden e na área 2, Furo Grande

\section{Fecundidade}

O comprimento das fêmeas ovadas variou entre $2.33 \mathrm{~mm}$ e $7.08 \mathrm{~mm}$. A fecundidade de 300 fêmeas foi observada: o número de ovos por fêmea variou entre 5 e 39 . Não foi identificada nenhuma correlação significativa entre o número de ovos $\mathrm{e}$ o tamanho das fêmeas ovadas $(r=$ $0.235 ; \mathrm{N}=300 ; \mathrm{P}>0.05$ ) (Fig. 5).

\section{Tamanho da primeira desova}

O tamanho teórico da primeira reprodução foi estudado considerando exclusivamente as fêmeas ovadas sem observar o aspecto dos ovários nem o diâmetro dos ovócitos. Um total de 1042 fêmeas ovadas foram examinadas, sendo 131 provenientes da área I, e 931 provenientes da área 2 (Fig. 6).

Calculou-se a percentagem das fêmeas ovadas em cada classe de comprimento, e em ambas as áreas, a percentagem de fêmeas ovadas apre- sentou uma distribuição bimodal: um número reduzido de individuos está maduro com comprimento pequeno $(2.25-4.0 \mathrm{~mm})$, enquanto a maioria das fêmeas ovadas apresentou um comprimento entre 5.0 e $7.0 \mathrm{~mm}$.

Considerando exclusivamente os individuos do grupo de maior tamanho, a reta de regressão ajustando a relação apresenta a forma linear seguinte:

$\%$ fềmeas ovadas $=43.93 \mathrm{Cc} \mathrm{mm}$ - $199.16(\mathrm{r}=0.96 ; \mathrm{N}=10)$ na área 1 , $\%$ fêmeas ovadas $=42.27 \mathrm{Cc} \mathrm{mm}$ $171.21(\mathrm{r}=0.94 ; \mathrm{N}=10)$ na área 2.

A estimativa do tamanho teórico da primeira desova é a interseção destas retas de regressão com o eixo de abscissa, ou seja:

$\mathrm{Lc}^{\circ}=4.53 \mathrm{~mm}$ na área $1 \mathrm{e} \mathrm{Lc}^{\circ}$ $=4.05 \mathrm{~mm}$ na área 2.0 tamanho teórico da reprodução maciça, quando $50 \%$ das fêmeas da população estão ovadas é de $5.67 \mathrm{~mm}$ na área 1 e de 


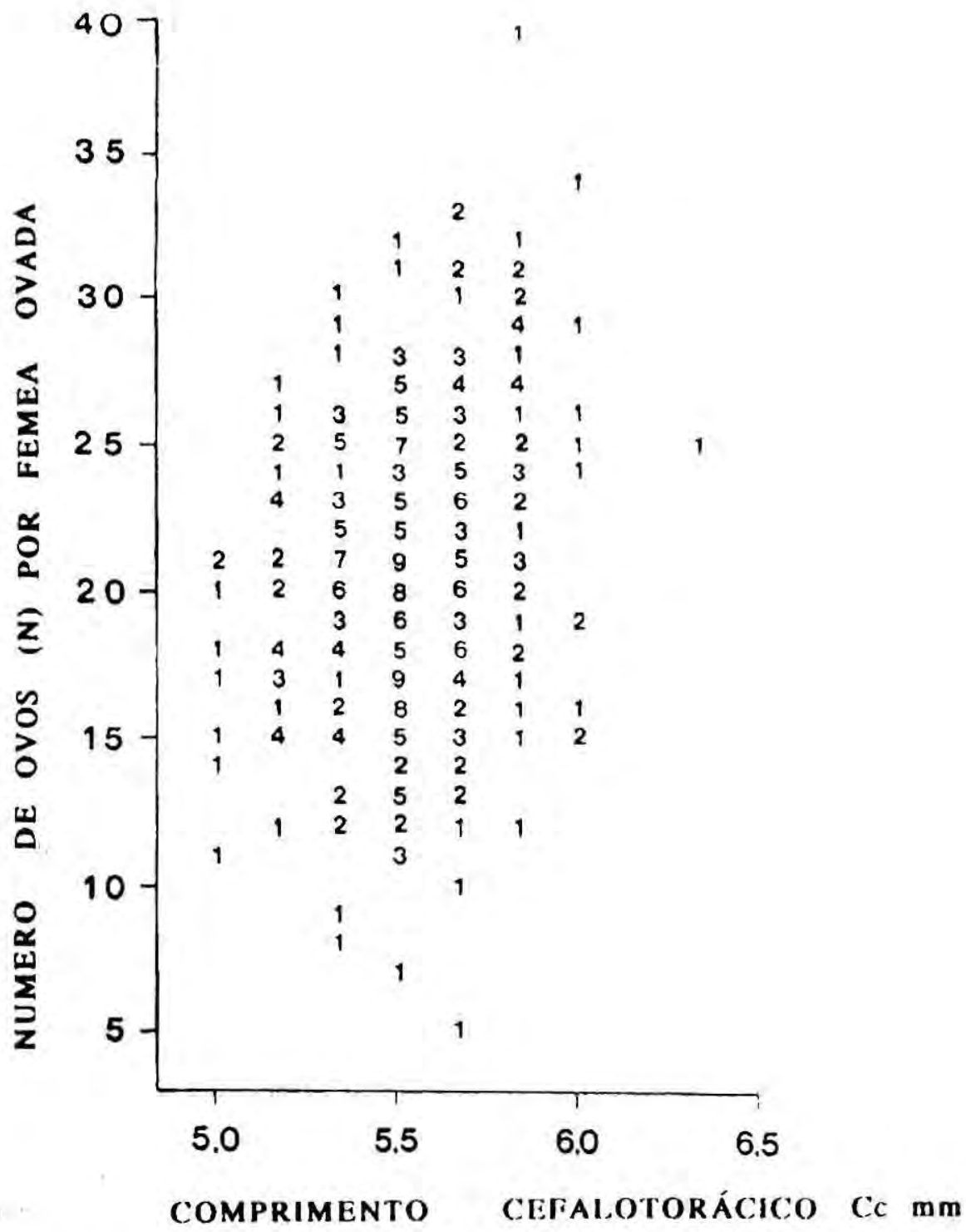

Figura 5. Fecundidade de $P$ carteri: frequências absolutas do número de ovos em linçũo do comprimento cefalotorácico de cada fêmea ovada. $(\mathrm{N}=300)$ fêmeas observadas; $\mathrm{r}=0.235 ; \mathrm{p}$ $0.05)$. 

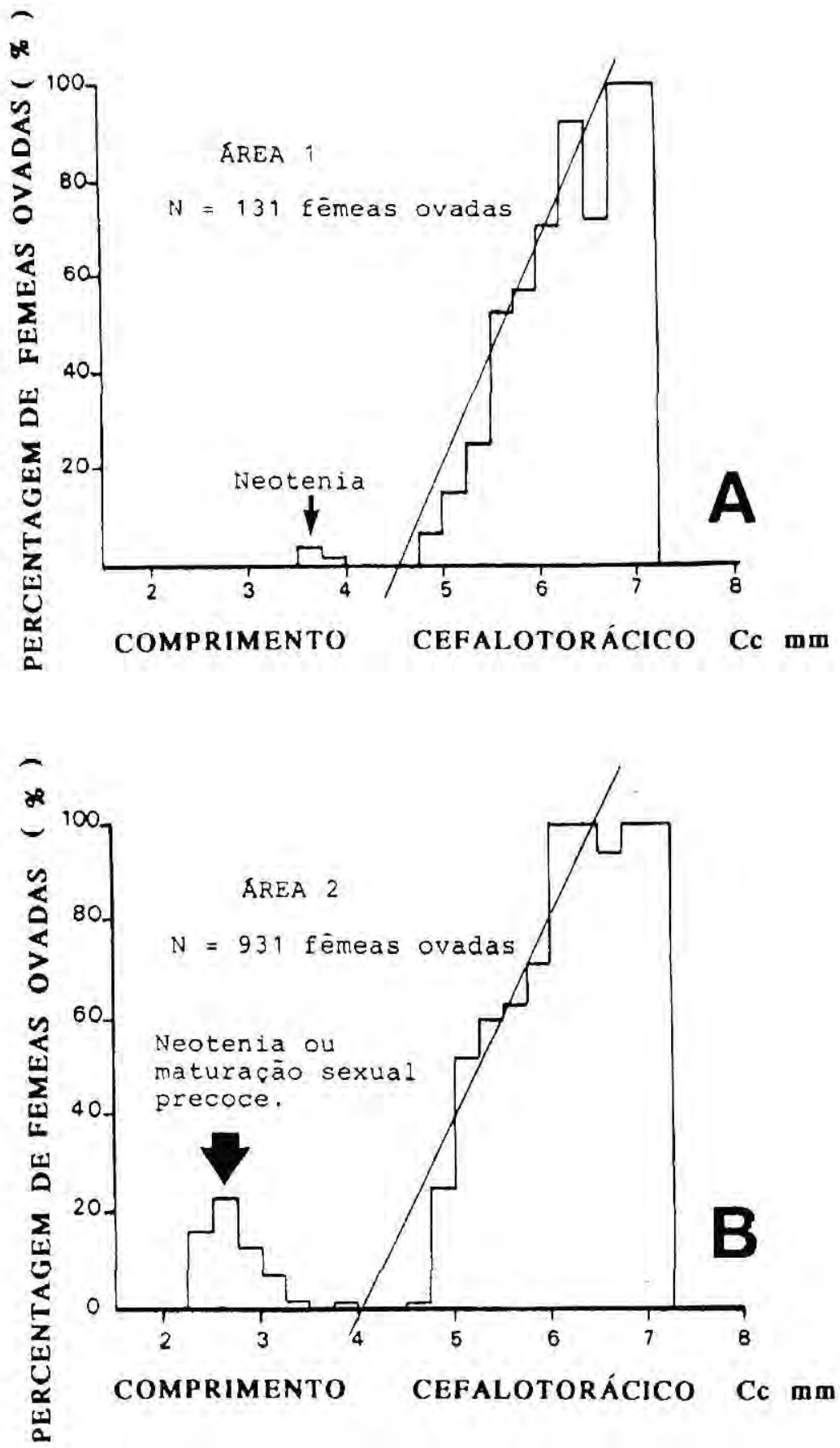

Figura 6. Evolução da percentagem de fềmeas ovadas de $P$. carteri em função do comprimento cefalotorácico, para cada área de coleta. 


\section{$5.23 \mathrm{~mm}$ na área 2.}

\section{Época de reprodução}

P.carteri apresentou uma reprodução sazonal bem marcada relacionada com o ciclo hidrológico. Em 1989, a duração do período de reprodução, determinado considerando a presença de fêmeas ovígeras na população, foi de sete meses. As fêmeas ovadas de P.carteri apareceram em ambas as áreas durante a enchente das águas, de janeiro até julho 1989 e à partir de fevereiro 1990. Elas foram ausentes nas coletas de agosto até janeiro de 1990, época da cheia, vazante e seca na Amazônia Central (Fig. 7 A e B).

Ovários em maturação (estágios 3 e 4) foram observados de janeiro até julho, assim como em proporção menor de outubro até dezembro em 1989, e em proporção maior a partir de fevereiro em 1990. A maioria das fềmeas apresentaram ovários imaturos de julho até fevereiro (Fig. 8).

\section{DISCUSSÃO}

\section{Caracterização sistemática.}

Palaemonetes carteri foi descrita por HOLTHUIS (1952) com os seguintes caracteres: 5 à 8 dentes na margem superior do rostro, e 3 à 7 dentes (geralmente 5 ou 6) na margem inferior; 0 espinho branquiostegal ultrapassando a margem anterior do cefalotórax. $\mathrm{O}$ estudo das populações do rio Negro mostra uma variabilidade semelhante do número dos dentes. Entretanto, cerca de $94.8 \%$ dos indivíduos apresentam uma variação bem menor, o rostro sendo ce terizado por 6 à 8 dentes na margem superior e 3 à 5 dentes na margem inferior. A localização do espinho branquiostegal não parece ser um carácter válido para descrição taxonômica desta espécie, jà que ela apresentou diversos posições, a mais frequente sendo no mesmo nível que a margem anterior da cefalotórax. Esses carácteres mostram uma grande semelhança entre as populações de P.carteri do rio Negro e a espécie irmã, Palaemonetes ivonicus, descrita do rio Beni na Bolivia (HOLTHUIS, 1950) e coletada em abundância na Amazônia central na várzea do sistema Solimões (HOLTHUIS, 1966; MAGALHAES, 1986a). Essas observações mostram a necessidade de analizar uma série de individuos em várias populações, sejam alopátricas, ou provenientes de hidrossistemas diferentes (lagos, igarapés, ria-lake, com água clara, preta ou branca) para descrever o padrão de variação dos caracteres morfológicos de uma espécie.

\section{ambientais.}

Os Palaemonidae apresentam uma grande diversidade de estratégias reprodutivas, que vão de uma metamorfose completa com eclosão de milhares de larvas zoé até o desenvolvimento direto de um número reduzido de juvenis (IHERING, 1897; SOLLAUD, 1923; GURNEY, 1942; WILLIAMSON, 1982; MAGALHÃES e WALKER, 1988 ). A fecundidade, o tamanho dos ovos e das larvas, assim como o número de estádios larvais variam dependendo das caracteristicas 


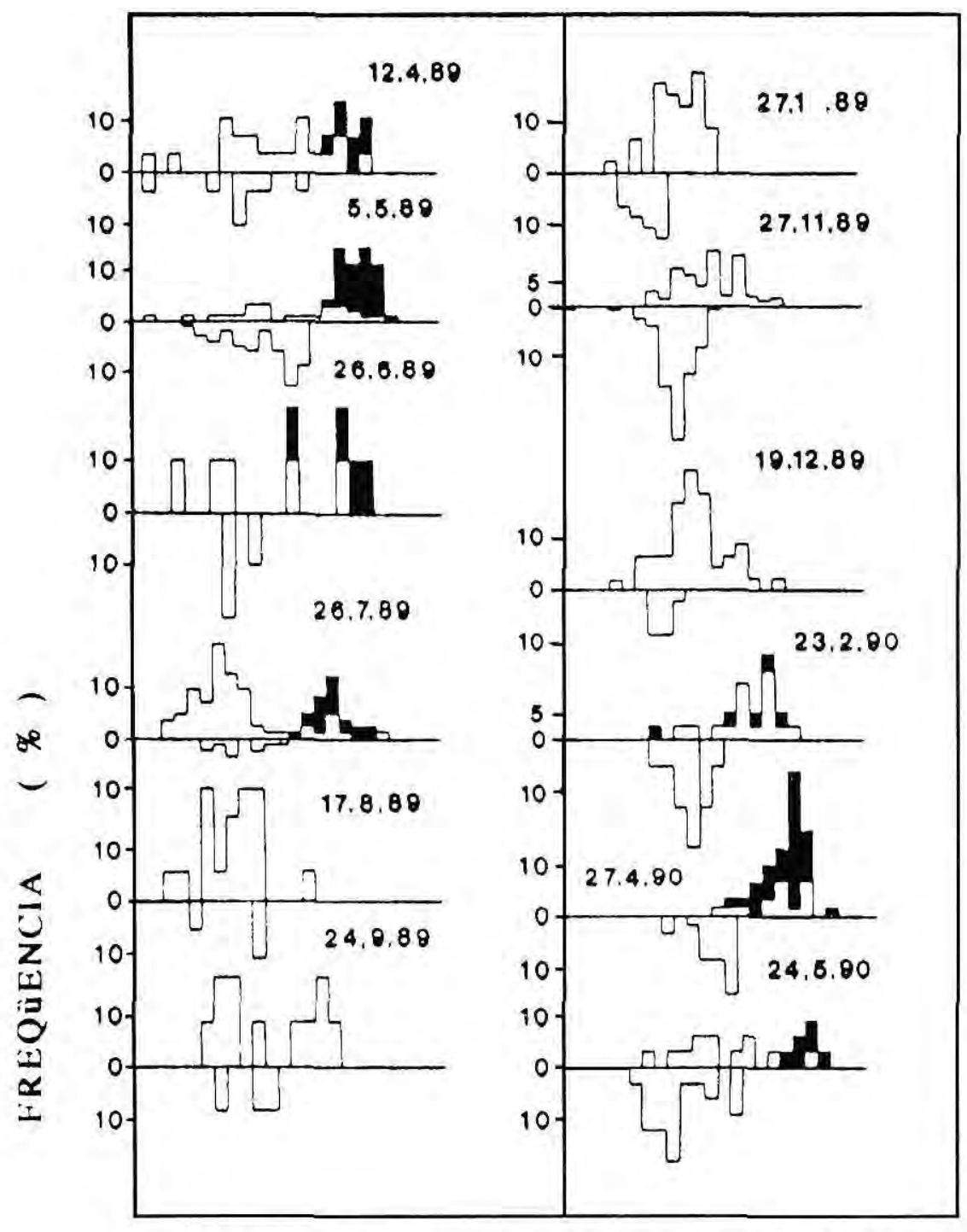

\section{COMPRIMENTO CEFALOTORÁCICO CC $\mathrm{mm}$}

Figura 7. Evolução sazonal da distribuição das frequências de comprimento de $P$ carteri coletado: a) no baixo Tarumãzinho (área 1) e b) no Furo Grande (área 2). As fêmeas são representadas acima do eixo (fêmeas ovígeras em preto) e os machos em baixo do eixo. 


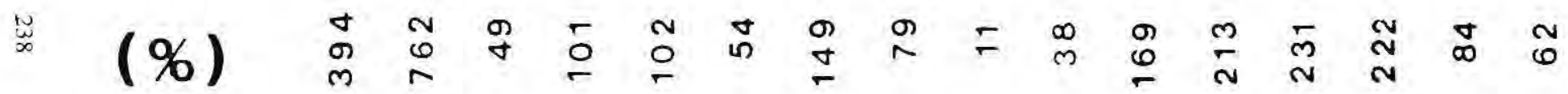
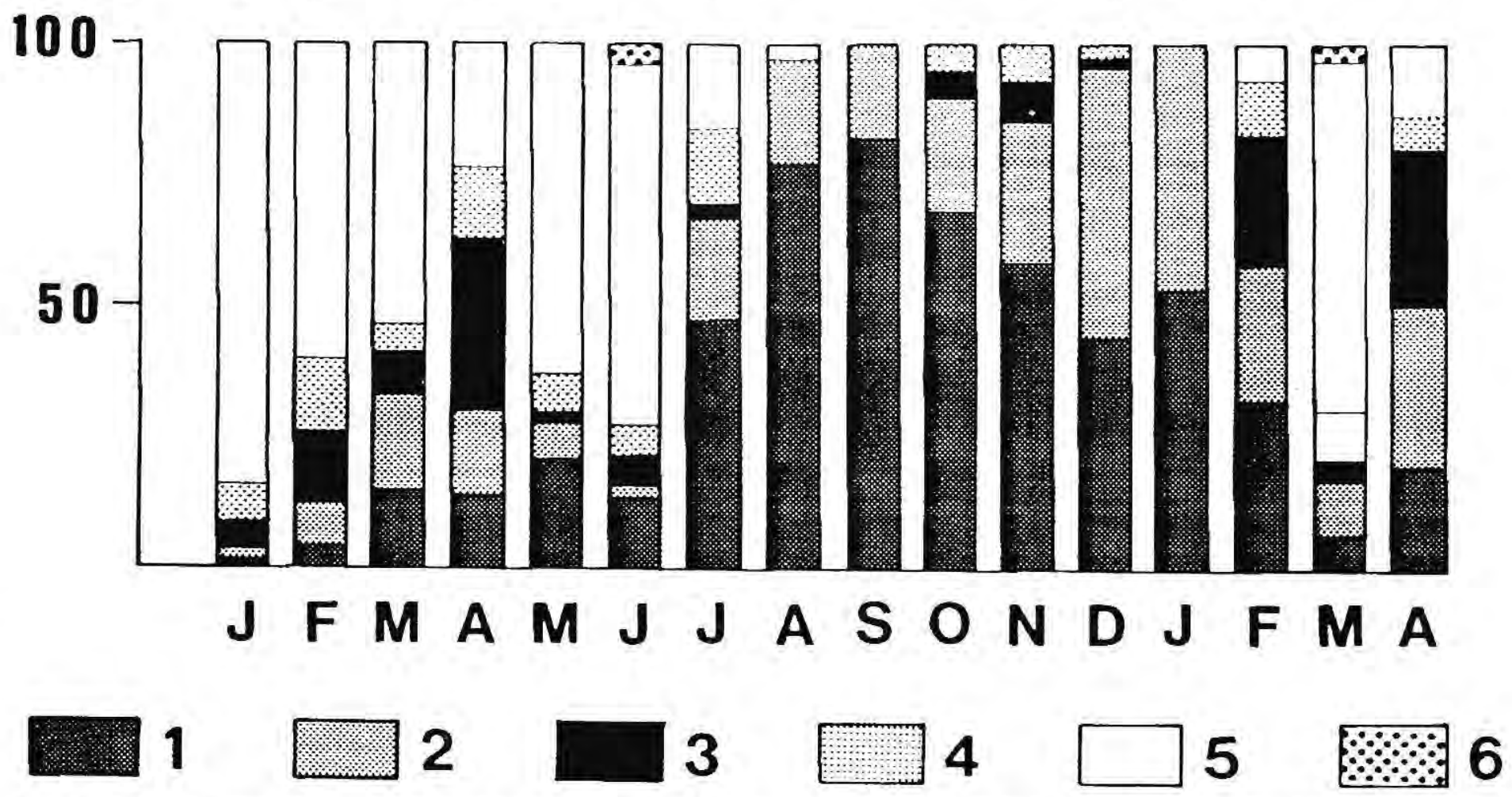

Figura 8. Variação mensal da frequencia relativa dos vários estádios de maturação ovocitária de $P$. carteri do rio Negro. As amostras das duas areas de coletada foram reagrupadas 
ecológicas do meio aquático. As espécies das águas continentais, de modo geral, desenvolveram uma estratégia reprodutiva caracterizada por um desenvolvimento abreviado e um número reduzido de ovos de tamanho grande, enquanto as espécies das áreas litorâneas apresentam metamorfoses completas, com larvas zoé planctônicas, cujo desenvolvimento é dependente das águas salobras dos estuários (WILLIAMSON, 1972; SHOKITA, 1973; JALIHAL \& SANKOLLI, 1975; POWEL, 1979; GAMBA, 1980; MAGALHÃES, 1985, 1986a, 1986b, 1988a, 1988b, 1989; MAGALHÃES \& WALKER, 1988; WALKER, 1992).

O número de ovos produzidos pelas fêmeas de uma determinada espécie, apesar de ser uma característica específica, pode variar em função da idade e do tamanho do indivíduo, assim como dos fatores ambientais como a oferta alimentar ou a temperatura. $\mathrm{Na}$ bacia Amazônica, Macrobrachium surinamicum e $M$. amazonicum. palaemonideos com metamorfoses completas, apresentam uma correlação significativa entre o número de ovos e o tamanho das fêmeas (ODINETZ COLLART, 1992; ODINETZ COLLART \& MAGALHÃES, no prelo). Apesar de mostrar diferentes padrões sazonais de reprodução, as várias populações de M.amazonicum seguem a mesma relação de fecundidade, sugerindo que os fatores filogenèticos sejam os principais fatores de controle de fecundidade em espécies com afinidade marinha. Ao contrário, as espécies continentais com desenvolvimento larval abreviado, como $M$. nattereri, P.carteri, Euryrhynchus amazoniensis ou $E$. burchelli, típicas de igarapés de terra firme, não mostram nenhuma relação entre o número de ovos e o tamanho da fêmea (ODINETZ COLLART \& ENRICONI, 1992; ODINETZ COLLART et al., 1992; ENRICONI \& ODINETZ COLLART, 1992). Em águas pobres em nutrientes e consequentemente em plâncton, fatores ecológicos como a oferta alimentar ou a densidade populacional, poderiam controlar não só o tipo de desenvolvimento larval mas a própria fecundidade à nivel do individuo (ODINETZ COLLART \& MAGALHÃES, 1992).

A discontinuidade de tamanho entre os dois grupos de fêmeas ovadas sugere um fênomeno de progênese, ou seja de maturação sexual precoce em indivíduos que ainda não apresentam todas as cara terísticas de adultos. Fenômeno de progênese não foi relatado em outras espécies de Palaemonídeos da Amazônia. Entretanto, HOLTHUIS (1952) observa que o estudo da taxonomia do gênero Macrobrachium pode ser dificultado por "indivíduos serem sexualmente maduros antes de desenvolver plenamente todas as partes do corpo. Por exemplo, fềmeas com caracteres juvenis podem já carregar ovos.."

A maioria dos palaemonídeos apresentam um padrão sazonal de reprodução. Em hidrossistemas tropicais, a maturação das gônadas e a época de reprodução estão principalmente controlados pelo regime hidrológico, através de fatores fisico-quimicos ou da correnteza 
da água. $M$. amazonicum apresenta uma reprodução continua na várzea onde as águas ricas em nutrientes garantem alimentação o ano todo. Palaemonídeos de igarapés de terra firme são caracterizados por um periodo de reprodução restrito à enchente das águas em áreas alagadas anualmente (WALKER \& FERREIRA, 1985; ODINETZ COLLART \& ENRICONI, 1992; ODINETZ COLLART et al., 1992; ENRICONI \& COLLART, 1992), enquanto eles se reproduzem o ano todo em igarapés permanentes de áreas mais altas (KENSLEY \& WALKER, 1982). Palaemonetes carteri segue o mesmo padrão, desovando durante a enchente e a cheia. Entretanto uma proporção pequena da população foi observada com ovários em maturação na seca. Um padrão semelhante foi observado em $M$. amazonicum que, apesar de se reproduzir o ano todo, apresenta picos reprodutivos no meio da enchente e da vazante, quando a velocidade da água está máxima, e na seca durante o pico de produção de fitoplâncton. A freqüência de fêmeas de $P$. carteri maduras aumenta de outubro para novembro, mas diminue em dezembro. Nenhuma fêmea ovada foi observada até fevereiro, quando começa a reprodução da população. Esses resultados sugerem uma regressão dos ovários e uma reabsorção dos ovócitos maduros por falta de oviposição. Ciclos de vitelogênese seguidos por reabsorção foi observado em vários decápodos (SASTRY, 1983). Em Jasus lalandii, este fenômeno resulta da ausência de fertilização (HEYDORN,
1969); em Homarus, ele aparece em condições ambientais desfavoráveis durante o periodo previsto de oviposição (AIKEN \& WADDY, 1980). Regressão de ovários maduros de $P$. carteri na ausência de fertilização foi observado em condições de laboratório (MAGALHÃES, com. pess.).

A predominância de fêmeas nas amostras de junho à setembro, durante o pico da reprodução e o início do recrutamento, pode ser interpretada por uma capturabilidade maior das fêmeas ovígeras e pós-desovadas, fenômeno frequentemente observado em camarões Palaemonídeos (ODINETZ COLLART, 1987; ODINETZ COLLART \& ENRICONI, 1992; ODINETZ COLLART et al., 1992).

Desovar durante a enchente é uma estratégia reprodutiva muito comum em espécies aquáticas tropicais, o que favorece a dispersão larval longitudinal para jusante, ou lateral nas planícies de inundação (WELCOMME, 1985). A população de $P$. carteri do rio Negro mostra uma oscilação sazonal dos comprimentos médios com valores superiores à média anual durante a enchente, época de reprodução da população, e valores inferiores durante a vazante e a seca, época do recrutamento. Este padrão reprodutivo tem um valor adaptativo em ambientes marcados por flutuações hidrológicas. Colonizar um habitat em expansão diminue a competição intraespecífica assim como a pressão de predação, aumentando conseqüentemente o fittness da própria espécie. 


\section{Bibliografia Citada}

AIKEN, D. E.; WADDY, S. L. 1980. Reproductive biology. In: J.S.COBB \& B.F.PHILLIPD (eds), The biology and managment of lobsters. Academic Press, vol 2, chap 4:215-276.

COELHO, P. A.; RAMOS-PORTO, M. 1985. Camarões de água doce do Brasil: distribuição geográfica. Rev. Bras. Zool. l(1):51-53.

ENRICONI, A.; ODINETZ COLLART, O. 1992. Ecologia do camarão Palaemonetes carteri na Amazônia Central, Rio Negro. In: Anais do $19^{\circ}$ Congresso Brasileiro de Zoologia. Belém, Brasil. 26-31 julho 1992.

GAMBA, A. L. 1980. Desarollo larval abreviado del camaron de agua dulce Macrobrachium jelskii (Miers, 1877). Mem. del Simp. Commemorativo $10^{\circ}$ Aniversario U.S.B. Caracas, Venezuela,pl69-189.

GOMES-CORREA, M. M. 1977 Palaemonideos do Brasil (Crustacea. Decapoda, Natantia). Dissertação de Mestrado. Universidade Federal do Rio de Janeiro. 135 p. 28 pls.

GURNEY, R. 1942. Larvae of Decapod Crustacea. Ray Society, London, 129:i-vi, 1306.

HEYDORN, A.E.F. 1969, Noteson the biology of Palinurus homarus and on length/ weight relationships of Jasus lalandii. Invest. Rep.Div.Sea Fish S.Afr, 69:1-27.

HOLTHUЛ, L. B, 1950. Prcimminary descriptions of twelve new species of Palaemonid prawns from american waters (Crustacea Decapoda). Proc. Kon. Nederl. Akad. Wetensch., 53:93-99.

HOLTHUIS, L. B. 1952. A general revision of the Palaemonidae (Crustacea. Decapoda, Natantia) of the Americas. II The subfamily Palaemoninae. Occ. Pap. Allan Hancock Found., 12:1-396, pls.1-55.

HOLTHUIS, L. B. 1966. A collection of freshwater prawns (Crustacea Decapoda,
Palaemonidae) from Amazonia, Brazil, collected by Dr. G. Marlier. Bull. Inst. rech. Sci. Nat. Belg. 4310

IHERING, H. von. 1897. Os camarões de água doce do Brasil. Rev. Mus. Paulista, 2:421-432.

JALIHAL, D. R., SANKOLLI, K. N. 1975. On the abbreviated metamorphosis of the freshwater prawn Macrobrachium hendersodayanum (Tiwari), in the laboratory. Karnatak Journ.Sci., 20:283-291.

KENSLEY, B.; WALKER, I. 1982. Palaemonid shrimps from the Amazon Basin, Brasil (Crustacea:Decapoda: Natantia). Smithsonian Contributions to Zoology, 362.

MAGALHÃES, C. 1985. Desenvolvimento larval obtido em laboratório de palaemonidos da Regiao Amazonica. I. Macrobrachium amazonicum (Heller, 1862) (Crustacea. Decapoda). Amazoniana, 9(2):247-274.

1986a. The larval development of palaemonid shrimp from the Amazon region reared in the laboratory. IV. Abbreviated development of Palaemonetes wonicus Holthuis, 1950 (Crustacea, Decapoda). Amazoniana, 10(1):63-78.

1986b. The larval development of palaemonid shrimp from the Amazon region reared in the laboratory. IV. Abbreviated development of Pseudopalaemon chryseus Kensley \& Walker, 1982 (Crustacea: Decapoda: Palaemonidae). Acta Amazonica, 16/17:95-108.

1988a. The larval development of Palaemonid shrimps from the Amazon region reared in the laboratory: II. Extremely abbreviated larval development in Euryrhynchus Miers, 1877 (Decapoda, Euryrhynchinae). Crustaceana 55(1):39-52.

1988a. The larval development of Palaemonid shrimps from the Amazon region reared in the laboratory: III. Extremely abbreviated larval development of Palaemonetes (Palaemonetes) mercedae Pereira, 1986 (Crustacea, 
Decapoda). Studies on neotropical fauna and environment, 23(1):1-8.

1989. The larval development of Palaemonid shrimps from the Amazon region reared in the laboratory: VI. Abbreviated larval development of Macrobrachium nattereri (Heller, 1862) (Crustacea, Decapoda). Amazoniana, 10(4):379-392.

MAGALHÃES FILHO, C; ; WALKER, I. 1988. Larval development and ecological distribution of central amazonian Palaemonid shrimps (Decapoda, Caridae). Crustaceana, 55(3):279-292.

ODINETZ COLLART, O. 1992. Ecología e potencial pesqueiro docamarão-canela, Macrobrachium amazonicum, na bacia amazônica.In:Bases cientificas para estratégias de preservação e desenvolvimento da Amazônia. (ed.) Ferreira, E.J.,; Santos, G. M.; Leão, E. L. M.; Oliveira, L. A., 2: 147-166.

ODINETZ COLLART, O.; MOREIRA, L. C. 1989. Quelques caractéristiques physico-chimiques d'un lac de varzéa en Amazonie centrale (Lago do Rei, île de Careiro). Rev. Hydrobiol. trop., 22(3):191-199.

ODINETZ COLLART, O; ENRICONI, A. 1992. Ecologia do camarão Macrobrachium natereri nos igarapés do Rio Negro, na Amazônia Central. In: Anais do $4^{\circ}$ Congresso Brasileiro de Limnologia. Manaus, Brasil.7-11 setembro 1992.

ODINETZ COLLART, O.; MAGALHÃES, C. 1992. Ecological constraints and life history strategies of Palameonid prawns in Amazônia.In: $X X V$ SIL International Congress. Barcelona, Spain. 21-27 août 1992.

ODINETZ COLLART, O; MEDEIROS, N.; ENRICONI, A. 1992. Cò-existência e estratégia reprodutiva de Euryrhynchus amazoniensis e $E$. burchelli nos igarapés do Rio Negro.In: Anais do $19^{\circ}$ Congresso Brasileiro de Zoole gia. Belém,Brasil, 2631 julho 1992.

PEREIRA, S. G. 1986. Freshwater shrimps from Venezuela I: seven new species of
Palaemonidae (Crustacea: Decapoda: Palaemonidae). Proc, Biol. Soc. Wash., 99(2):198-213.

POWELL, C. B. 1979. Suppression of larval development in the African freshwater shrimp Desmocaris trispinosa (Decapoda, Palaemonidae). Crustaceana, (suppl,) 5:185-194.

RODRIGUEZ, G. 1981. Decapoda. In: Aquatic Biota of Tropical South America, Part I: Arthropoda. S. H. HURLBERT, G. RODRIGUEZ, N. D SANTOS (eds.). San Diego State University, San Diego, California.

RODRIGUEZ, G. 1982. Freshwater shrimps (Crustacea, Decapoda, Natantia) of the Orinoco Basin and the Venezuelan GuayanaJ. Crust.Biol., 2 (3): 378-391.

SASTRY, A. N. 1983. Ecological aspects of reproduction. In: The Biology of crustacea. D.E. BLISS (ed.), 8(5): 179-270.

SHOKITA, S. 1973. Abbreviated larval development of the fresh-water prawn, Macrobrachium shokitai Fujino et Baba (Decapoda, Palaemonidae) from Oriomote Island of the Ryukyus. Annot. zool. Japon., 46(2):111-126.

SOLLAUD, E. 1923. Le développement larvaire des "Palaemoninae". I.Partie descriptive. La condensation progressive de l'ontogénèse. Bull. Biol. France Belgique, 57: $509 \cdot 603$

SUBRAHMANYAM, C. B, 1965. On the unusual occurence of Penaeid eggs in the inshore waters of Madras. J. mar. biol. Ass. India., 7 (1):83-88.

WALKER, I. 1992. Life history traits of shrimps (Decapoda: Palaemonidae) of amazonian inland waters and their phylogenetic interpretation. Studies on neotropical fauna and environment, 27(2/3):131-143.

WALKER, I; FERREIRA, M. J. N. 1985. On the population dynamics and ecology of the shrimp species (Crustacea, Decapoda, Natantia) in the Central Amazonian river Taruma-Mirim. Oecologia, 66:264-270. 
WELCOMME, R. L. 1985. Rivet Fisheries.

F.A.O. Fish. Tech. Pap, (262): 330 p.
WILLIAMSON, D. 1. 1972. Larval developent in a marine and a freshwater species of Macrobrachium (Decapoda, Palaemonidae). Crustaceana, 23: 282 298. 